\title{
Biomechanical and kinesiological analysis of the jumpmovement and landing in the volleyball slash
}

DOI: $10.46932 / \mathrm{sfjdv2n2-088}$

Received in: March 1st, 2021

Accepted in: May 30th, 2021

\section{Prof. Esp. Célio De Arruda Camargo Junior}

Especialista em Voleibol, pela FIG - Faculdades Integradas de Guarulhos, 1992 Instituição: USA South Volleyball Center

Endereço: 19980 Barletta Ln 921, Estero - FL, USA

Zip code 33928-5670

E-mail: celioarruda@yahoo.com.br

Prof. Me. Celso Luiz Bastos

Mestre em Educação, pela Universidade Cidade de São Paulo, 2003 Instituição: Faculdades Metropolitanas Unidas

Endereço: Rua São Jorge, 300, AP. 184, Parque São Jorge - São Paulo, SP, Brasil -CEP: 03087-000

E-mail: celso.bastos@uol.com.br

\section{Prof. Dr. Frank Shiguemitsu Suzuki}

Doutor em Educação Física pela Universidade São Judas Tadeu, 2016 Instituição: Universidade Nove de Julho

Endereço: Rua Tamaindé, 1055, ap, 63, Vila Nova Manchester, São Paulo, SP, Brasil -CEP: 03444-000 E-mail: profsuzuki@uni9.pro

\section{ABSTRACT}

Volleyball is a sport Best practiced when its gestures are analyzed under the concepts of Biomechanics. So, we decided to develop a biomechanical study about the movements used for hitters/attackers at position 4 . The study was carried out by analyzing photos and graphs of the execution of this movement presented in scientific articles, attempting to determine if there exists a prime angle (of both upper and lower limbs) foreach phase of the gesture (approach, impulse, airtime, and fall).

Keywords: biomechanics in volleyball, study of cutting in volleyball, kinesiology in attack in volleyball

\section{INTRODUCTION}

The practice of guided physical exercise, every day more, becomes indispensable to the global development of the human being, and Sports is one of the components that most attracts the various age groups.

In the last decades, Volleyball has been occupying a prominent place amongour young people, especially those who propose to enjoy the biopsychosocial benefits that the sport presents.

From the interest for the practice of this sport, motor learning, specific physical exercise, and group coexistence, fundamental characteristics of volleyball, will lead to further improvement and the 
search for information that will meet the expectations of the interested party.

With the concern in providing an efficient and effective learning, with the least possibility of injury acquisition, through a practice that has little scientific basis in the performance of physical-motor tasks, we propose to present a study that approaches some phases of the CORTADA movement under the concepts of biomechanics and kinesiology, which will support a proposal for the performance of this motor action, pointing out the best indications and advantages for its execution, with the best use of their physical abilities and skills.

We notice that several methods are presented for the best use of the cut, whether in the physical, motor, psychological, or biomechanical aspects, because it is through this skill that the highest number of points is obtained during a volleyball match.

When we analyze a specific motor skill (technique) in sport, we must take into account several factors involved, among them, the mechanical action. It is with this objective that we intend to discuss a more efficient form of movement, relating the specific technique of the sport with the biomechanics used to obtain the optimization of this movement. The interaction of these factors provides, to the athletes, the efficacy in the execution, as well as a greater longevity in their career, because we believe that there will be a decrease in injuries, derived from the inadequate execution of the slicingmovement.

To be more specific, we will talk about athletes who play in position 4 , in thefront line (called "POINTERS") and who receive the greatest amount of attacked balls during a match

To be more specific, we will talk about the athletes who play in position 4 , on the front line (called "PONTERS") and who receive the most balls attacked during a match. In this position (4) and function (top striker), the physical demands have specific characteristics: great dynamic vertical thrust, muscular power of the attack arm(in this case, the right arm), and greater motor dexterity (overcoming the opponent's blockade).

Therefore, the possibilities of execution of the proposed movement (CORTADA) will receive more attention in its definition.

All phases of the CORTADA will be considered:

1- Approach or Acceleration;

2- Jump or take-off;

3- Air phase

4- Landing (fall).

Considering the perfect pass (between positions $2 / 3$ ), the lifter will be in front of the attacker, near the net, in a regular distance (adequate to his/her individual characteristics), or normal, for the 
execution of the attacking action.

$=$ striker $($ position 4$)$

$=\operatorname{lifter}($ position 2/3)
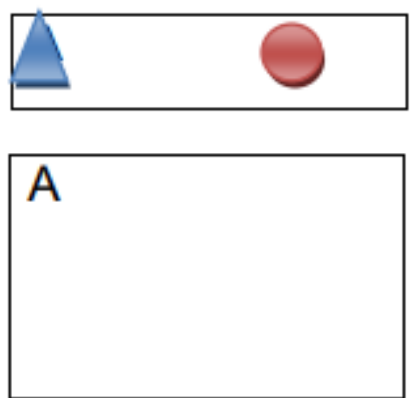

The attacker (A) will be in the initial position of his run, or approach to the ball, outside the court, remembering that there are many variations and concepts, that is why we are looking for a basic movement, which does not consider other schools (Asian, Russian, and others), and individual styles, because we consider them exceptions or optimized movements.

\section{OBJECTIVES}

To gather evidence, from the literature, about the biomechanics of the volleyball cuttingthrust and landing movements.

\section{METHOD}

The study was conducted by analyzing movement figures, which are presented in scientific articles. The key words used for the search were volleyball and biomechanical analysis. The search was made through the google academic site. 


\section{RESULTADOS:}

\subsection{DESCRIÇÃO DO MOVIMENTO ANALISADO}

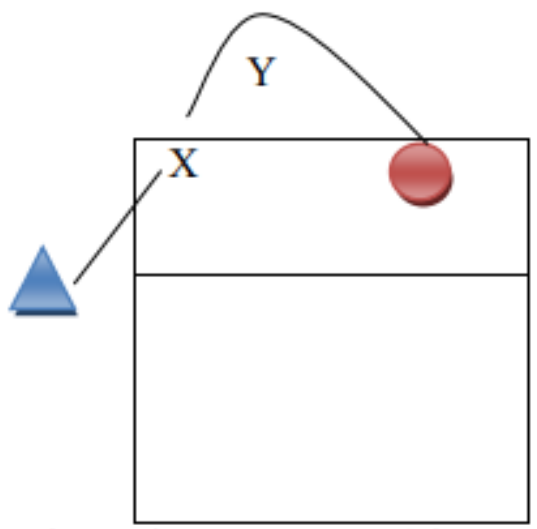

To begin the detailed description of the movement, we will first name where the ball will be lifted, which will be the estimated point of contact with the ball, rememberingthat when we attack in this position, contact with the ball is made on the downward trajectory.

Note: the trajectory of this lift (ball height + speed) can alter the athlete's "approach". The Y point represents the height, or contact point, which varies according tothe individual possibilities of each attacker (higher or lower).

The $\mathrm{X}$ point represents the ball's reference in relation to the net and the court's lateral line. This point is considered the ideal "POINT" for the athletes and it is recommended "one meter away from the net and sixty centimeters in relation to the side line of the court".

As in all volleyball fundamentals, we must guide the athletes how to move towards the ball and jump, what we usually call "approach", which is the combination of running and jumping towards the ball, with energy to reach it at the highest point and strike it with as much power as possible, towards the opponent's court, making the defensive action of the opposing team difficult, or impossible, and, most importantly, score points for their team

At this point, we will analyze the "approach" most used by beginners, that is, with three steps. The three-step approach is easier for beginners to learn because it requires lesscoordination for its execution. To begin the approach, we must determine the ideal startingand ending point for the takeoff, in the direction of the air phase Notice that the analysis ofthe "approach" requires a kinematic, physical, geometric, and biomechanical study, because we will analyze a body in motion

Our proposal is that by approaching this specific movement, that is, the cut performed in position 4 by right-handed athletes, we will facilitate the understanding of the "ADVANTAGES" that the movement may present, both for beginners and for more experienced athletes, and its biomechanical 
optimization for a greater effectiveness of this motor action.

Because we consider that the cutting movement contains 4 phases, as we will describe below, we will make the descriptions of each phase and their respectivecharacterizations (Figure below):
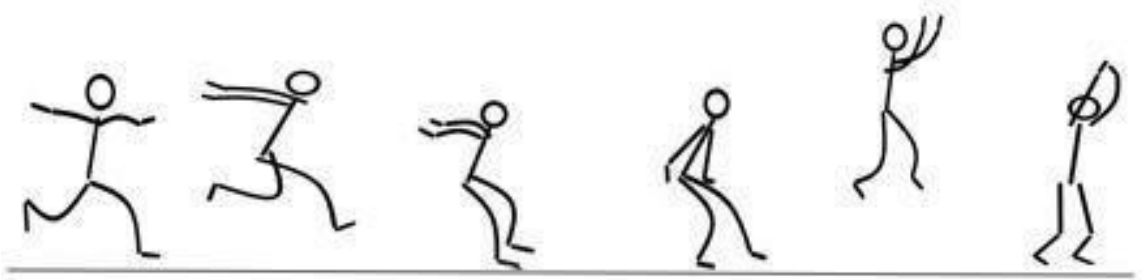

\subsubsection{Phase 1 = "approach" or approach and preparation for the jump towards theball.}

From the initial position, in a situation of starting acceleration, until the

"approach", some "postures" of body segments should be respected for the best use of the approach to the ball until the moment of "impulsion". In the case of a right-handed player, the position of expectation will be with the right foot in front, starting theapproach with the left foot, which we consider the first step. This step should be longer than shoulder distance, in a slow and straight line. The player should keep his eyes turnedtowards the hoist and consequently to the ball, facilitating his jump time or "ball time" (synchronism between the hoist and the contact with the ball).

Remember that as the approach is a combined movement between the upper limbs(arm swing) and the lower limbs (the stride). The athlete should not throw the arms abovethe chest line during the first stride, as this action will trigger a slower displacement, leading to a delay in the start of the second stride.

In this first step, we also identified players moving their arms alternately, as in a race, but beginners show greater ease in learning the movement with both arms thrown forward simultaneously.

In the second step, of immense importance, the athlete will perform a step with greater amplitude, with twice the distance of the first, and faster than the first.

To do so, the athlete will add a small air phase with the objective of gaining stride amplitude and generating a force against the ground that, when it contacts it, will result ingreater impulsion (Newton's third law - action and reaction).

At that moment, when you contact the ground with your heel, your arms will be thrown behind your torso and above your shoulders (to facilitate this action, we recommend moving your arms a little away from your torso, like a "wing" and directing the palm of your hand upwards). This swing of the arms behind and then in front of the body during the jump phase will provide a gain in impulsion of up to $23 \%$, since the armsrepresent $10 \%$ of body weight. 
In the third step, when contacting the ground with the tip of the left foot almost parallel to the net (with the goal of minimizing horizontal impulsion), the transition from running to jumping, or from horizontal to vertical speed begins.

At this moment, both arms will be thrown in front of the body, explosively and finishing above and in front of the head. This is the moment when we leave the ground,or take off initiating the aerial phase.

\subsubsection{Kinesiological and biomechanical analysis:}

The approach is nothing more than running toward the ball, that is, the linear and uniform displacement of the body dynamically. For this, the ankle, knee, hip, and spine joints must move dynamically and angularly in order for the body to move.

From position 1 to 2 , the glenohumeral joint is extended and promotes hyperextension. The right hip joint performs the joint flexion movement in concentric action using the hip flexor muscle group (Iliopsoas and Rectus femoris). From position 2 to 3, the right knee joint performs an extension in eccentric action and the left knee joint performs a flexion in concentric action using the knee flexor muscle groups (Ischiotibial and Medial and Lateral Gastrocnemius).

\subsubsection{Phase 2 - impulsion (jump)}

From that moment on, there will be a plantar flexion of the ankle joint and extension of the knee joint, and a hyperextension of the hip joint, followed by a hyperextension of the spinal joint, leading the body to create an image compared to an "arch". Therefore, it is possible to understand the difficulty of learning and performingthe movement, as it requires a series of movements in such a short time, as well as dynamic balance and spatial orientation.

The rhythmic combination of biomechanical and motor actions will provide enormous advantages to the performer, in terms of taking greater advantage of themuscular power necessary to reach the highest and best point of contact with the ball. At the moment of the jump, the trajectory changes as a function of the approach, so the displacement becomes vertical.

\subsubsection{Phase 3 - air}

Once in the air, and in an "arc" shape, we begin the asymmetrical coordinated movement between the right upper extremity, left upper extremity, and lower extremity. The next action is to throw the elbow of the attacking, or dominant, arm behind the shoulders and in line with them. This action can be compared, with some mechanical differences, to that performed by an archery athlete at the 
moment of "pulling the arrow" to generate energy for the shot."

In an asymmetric but synchronized action between the right and left arm, described as follows: while the attacking arm (right) rotates the glenohumeral joint and extends the arm towards the ball (generating almost $80 \%$ of the power of the cut), the left arm starts the downward movement, in a circular way and out of the trunk line, towards the hip and will rest in front of the body, with the elbow flexed at the hip joint.

At the moment of ball contact, we observe a straight line from the contact hand, tothe player's left foot, due to the elevation of the right shoulder and, consequently, the inclination of the spine.

The contact of the hand on the ball will be made, most of the time, with the fingers separated, or with the hand open and behind the ball (recommended for beginners or players with little range of attack, remembering that we are talking about a traditional sliceand not an emergency technique). After this movement, the palm of the hand will slide over the ball, with the objective of obtaining the "spin" of the ball, directing it to the ground. In the case of some elite players, they do not use this technique, because they jump very high and place their open hand, or not, on top of the ball.

The aerial phase of the movement is completed with the descent of the dominant, or attacking, arm in a perpendicular direction to the chest, finishing it in front with the legcorresponding to the arm.

\subsubsection{Phase 4 - landing (fall)}

The return to the ground is one of the least addressed issues in initiation, or evenin more experienced practitioners.

The first contact with the ground should occur with the forefoot, and then the midfoot, followed by the ball of the foot, and finally the hindfoot, and in the sequence flexion of the knee joints, slight flexion of the trunk forward, and the arms assist in the recovered balance.

The importance of landing is due to the great impact that our body suffers when it touches the ground, which, due to the force of gravity, will be two to three times our bodyweight.

When we observe a non-traditional landing, or different from what we described above, for example, falling on the support of only one foot, we know that this may have occurred by using an emergency technique, such as turning your whole body to the short diagonal and attacking the ball in parallel, or by unfamiliarity with the landing technique.

The landing moment is the most critical and damaging moment in the process, because we are generating a force of 2 to 3 times the normal body weight on the area of first contact with the ground. If we follow the steps described above, the first contact withthe forefoot, an overload will be generated on the calcaneal tendon. If the first contact is with the midfoot, it will generate plantar fasciitis problems. 
If the first contact is with the heel, all joints will suffer an excessive load (ankle, knee, hip, and spine joints), that is, there will always be some consequence.

\subsubsection{Phase 1 - the beginning of the acceleration or first step}

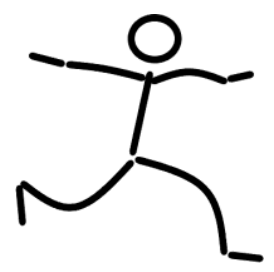

4.2.2 Phase 2 : The second step

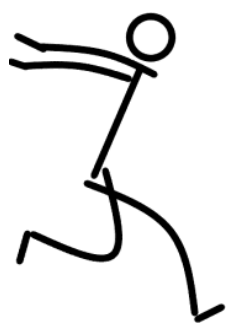

\subsubsection{Phase 3: the takeoff and air phase}
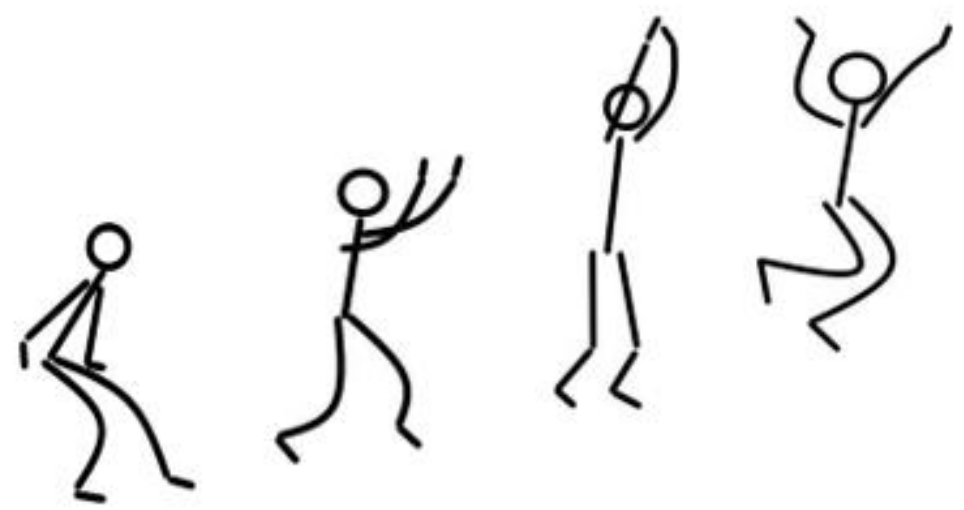


\subsubsection{Phase 4 - the landing}
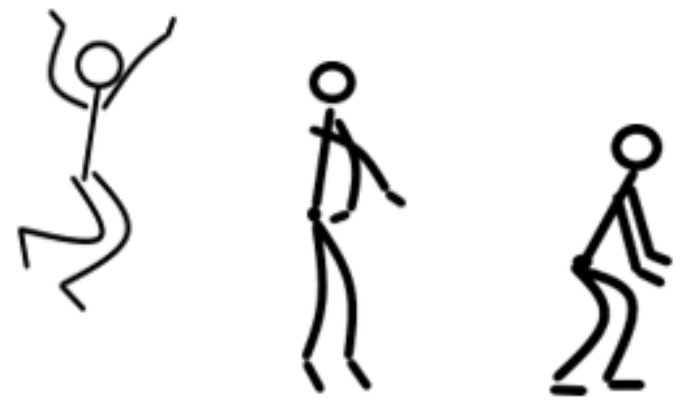

Chart :

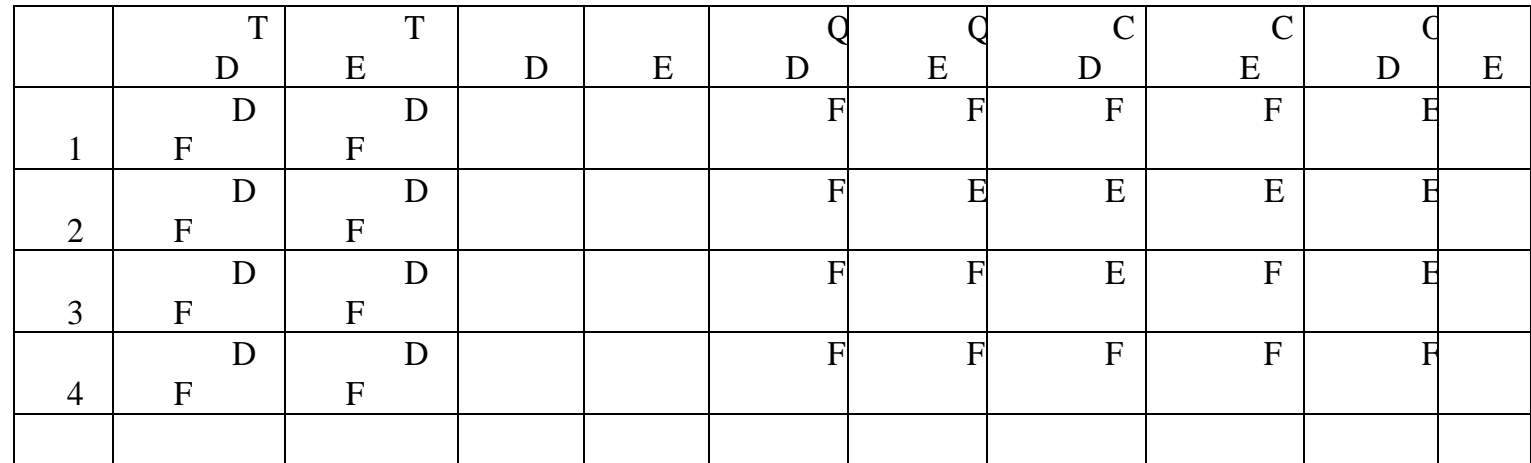

Subtitle:

TD=RIGHT ANKLE; TE=LEFT ANKLE; JD=RIGHT KNEE; JE=LEFT KNEE; QD=RIGHT HIP; QE=LEFT HIP; $\mathrm{CD}=$ RIGHT ELBOW; CE=LEFT ELBOW; OD=RIGHT SHOULDER; QE=LEFT SHOULDER.

F1=PHASE 1; F2=PHASE 2; F3=PHASE 3; F4=PHASE 4. DF=DORSIFLEXION; FP=PLANTARFLEXION; $\mathrm{E}=\mathrm{EXTENSION} ; \mathrm{F}=\mathrm{FLEXION}$

\section{FINAL CONSIDERATIONS}

Volleyball is clearly a high-impact sport, not only because of the numerous jumps,but also because of the execution of defense techniques such as rolling, diving, and others.

We do not propose to completely eliminate the small injuries caused by all these impacts, but if the player has excellent technique, or adequate biomechanics, in additionto physical preparation aimed at both injury prevention and high performance, this athlete will minimize the appearance of such injuries, and will be able to enjoy a longer career.

We must also focus on the aspects that will interfere in the performance of the jump, because genetics is a predominant differential, being responsible for seventy-five percent of the height reached in the jump. Anthropometry, which refers to the athlete's body measurements, can be a facilitating agent for gains in performance. The perfect use of all the muscles required in the jump, especially the gluteal muscles, which can generate up to forty percent of the impulsion. The levers involved in the jump, using the correct angles of the joints to maximize the jump. The acceleration of the steps, as well astheir 
size, and the final speed of the approach, which if done progressively, will add to theperformance gain.

Knowing all the biomechanical phases of the movement, the next step will be to detect problems in the execution of the shot, study the cause of the lack of performance, acause that may not be related to biomechanics, but to the specific technique of the sport, and bring a possible solution. For example: athletes who get under the ball a lot, usually start the approach too early, while athletes who attack the ball in the net frequently, usually contact the ball with their hand on the ball.

The result of the biomechanical and kinesiological analysis has the intention of better understanding each sport gesture, with the concern of preventing future appearance of injuries, due to inefficient movements. From the analysis, we can better understand the movements and direct the preparation of structures that will suffer the impacts of the sport. 